\title{
Qualidade de Vida Relacionada à Saúde de Mulheres com Câncer de Mama em Tratamento Radioterápico: Revisão Integrativa da Literatura
}

\author{
doi: https://doi.org/10.32635/2176-9745.RBC.2021v67n3.1264 \\ Health-Related Quality of Life of Women with Breast Cancer Undergoing Radiotherapy: Integrative Literature Review \\ Calidad de Vida Relacionada con la Salud de Mujeres con Cáncer de Mama Bajo Tratamiento con Radioterapia: Revisión \\ Integrativa de la Literatura
}

\author{
Lais Reis Siqueira'; Sterline Therrier²; Pabliane Matias Lordelo Marinho³; Camila Mendonça de Moraes ${ }^{4}$; Zélia Marilda Rodrigues \\ Resck $^{5}$; Sinézio Inácio da Silva Junior ${ }^{6}$; Namie Okino Sawada7
}

RESUMO

Introdução: $\mathrm{O}$ câncer de mama é a principal causa de morte por câncer em mulheres no Brasil. A radioterapia é uma modalidade terapêutica frequentemente utilizada no tratamento do câncer de mama. Apesar de pouco invasiva, causa efeitos colaterais, interferindo na Qualidade de Vida Relacionada à Saúde (QVRS). Objetivo: Investigar as evidências sobre a QVRS de mulheres com câncer de mama em tratamento radioterápico. Método: Trata-se de uma revisão integrativa da literatura. A busca dos estudos ocorreu no mês de agosto de 2020 nas bases eletrônicas de dados: CINAHL e MEDLINE (PubMed). Resultados: Os estudos analisados abordaram os efeitos colaterais do tratamento radioterápico e apresentaram evidências de práticas efetivas para amenizá-los e melhorar a QVRS. A amostra final foi constituída por 16 artigos, dos quais foram selecionadas três categorias que influenciavam a Qualidade de Vida: efeitos adversos da radioterapia (75\%); terapias integrativas e complementares (19\%); e atividade física (6\%). Conclusáo: Espera-se que a síntese dos estudos analisados forneça subsídios para a melhoria da prática clínica dos profissionais que prestam assistência às mulheres com câncer de mama em tratamento radioterápico.

Palavras-chave: Qualidade de Vida; Neoplasias da Mama; Mulheres; Radioterapia.

\section{ABSTRACT}

Introduction: Breast cancer is the leading cause of death by cancer in Brazilian women. Radiotherapy is a therapeutic modality frequently used in the treatment of breast cancer. Although little invasive, it causes side effects, interfering in the Health-Related Quality of Life (HRQoL). Objective: To investigate the evidence about the HRQoL of women with breast cancer undergoing radiotherapy treatment. Method: Integrative review of the literature. The search for studies took place in August 2020 in the electronic databases: CINAHL and MEDLINE (PubMed). Results: The studies analyzed addressed the side effects of radiotherapy treatment and presented evidence of effective practices to minimize them and improve the HRQoL. The final sample consisted of 16 articles, from which three categories which influenced the Quality of Life were selected: adverse effects of radiotherapy (75\%), integrative and complementary therapies (19\%) and physical activity (6\%). Conclusion: It is expected that the synthesis of the studies analyzed are instrumental to improve the professionals' clinical practice who provide care for women with breast cancer undergoing radiotherapy. Key words: Quality of Life; Breast Neoplasms; Women; Radiotherapy.

\section{RESUMEN}

Introducción: El cáncer de mama es la principal causa de muerte por cáncer en mujeres en Brasil. La radioterapia es una modalidad terapéutica de uso frecuente en el tratamiento del cáncer de mama, aunque poco invasiva, provoca efectos secundarios, interfiriendo en la Calidad de Vida Relacionada con la Salud (CVRS). Objetivo: Investigar la evidencia sobre la CVRS de las mujeres con cáncer mama sometida a tratamiento de radioterapia. Método: Se trata de una revisión integradora de la literatura. La búsqueda de estudios se realizó en agosto de 2020 en las bases de datos electrónicas: CINAHL y MEDLINE (PubMed). Resultados: La muestra final estuvo conformada por 16 artículos, de los cuales se eligieron tres categorías que influyeron en la Calidad de Vida: efectos adversos de la radioterapia (75\%); terapias integradoras y complementarias (19\%); y actividad física (6\%). Los estudios analizados abordaron los efectos secundarios del tratamiento con radioterapia y presentaron evidencia de prácticas efectivas para paliarlos y mejorar la CVRS. Conclusión: Se espera que la síntesis de los estudios analizados proporcione subsidios para mejorar la práctica clínica de profesionales que brindan asistencia a mujeres con cáncer de mama sometidas a radioterapia. Palabras clave: Calidad de Vida; Neoplasias de la Mama; Mujeres; Radioterapia.

\footnotetext{
1,25,5,7Universidade Federal de Alfenas (Unifal). Alfenas (MG), Brasil. E-mails: laisreis.siqueira@gmail.com; sterlinetherrier@gmail.com; zmrresck57@gmail.com; sinezio@unifal-mg.edu.br; namie.sawada@unifal-mg.edu.br. Orcid iD: https://orcid.org/0000-0002-6720-7642; Orcid iD: https://orcid.org/0000-0002-7713-3288; Orcid iD: http://orcid.org/0000-0002-3752-8381; Orcid iD: https://orcid.org/0000-0001-9385-9698; Orcid iD: http://orcid.org/0000-0002-1874-3481 ${ }_{3}^{3}$ Universidade Federal de Sergipe. Ribeirão Preto (SP), Brasil. E-mail: marinho.pabliane@gmail.com. Orcid iD: https://orcid.org/0000-0001-6190-0844 ${ }^{4}$ Universidade Federal do Rio de Janeiro (UFRJ). Rio de Janeiro (RJ), Brasil. E-mail: camila.moraes@unifal-mg.edu.br. Orcid iD: https://orcid.org/0000-0001-5544-8409 Endereço para correspondência: Lais Reis Siqueira. Unifal. Rua Gabriel Monteiro da Silva,700 - Parque das Nações. Alfenas (MG), Brasil. CEP $37130-001$. E-mail: laisreis.siqueira@gmail.com
} 


\section{INTRODUÇÃO}

O câncer de mama é o tipo mais comumente diagnosticado e a principal causa de morte por neoplasias entre as mulheres, sua incidência é superada apenas pelo câncer de pele não melanoma ${ }^{1}$. A escolha do tratamento para essa patologia é geralmente baseada nas características anatomopatológicas, estadiamento do tumor, idade, condiçôes clínicas, preferências do paciente ${ }^{2,3}$.

Entre as modalidades mais comuns de tratamento para câncer de mama aparece a radioterapia adjuvante, correspondendo a $49 \%{ }^{4}$. Apesar de pouco invasiva, a radioterapia causa efeitos adversos, entre os mais recorrentes estão: radiodermite, escurecimento da pele, prurido, descamação, dor local, desconforto axilar e fadiga. Tais sintomas interferem na saúde como um todo, afetando a parte física, emocional, psicossocial e consequentemente influenciando a Qualidade de Vida Relacionada à Saúde (QVRS) ${ }^{5-9}$.

A Qualidade de Vida (QV) é definida como: "a percepção do indivíduo de sua posição na vida, no contexto da cultura e sistemas de valores nos quais vive e em relação aos seus objetivos, expectativas, padróes e preocupações" ${ }^{10}$. Destaque que envolve aspectos multidimensionais relacionados ao bem-estar físico, bem como aspectos sociais, espirituais, psicológicos e subjetivos ${ }^{11}$.

Dessa forma, a pesquisa se deu com o intuito de sintetizar conhecimentos que possam fornecer subsídios para a melhoria da prática clínica dos profissionais que prestam assistência às mulheres com câncer de mama em tratamento radioterápico e consequentemente tenham um impacto positivo na sua QVRS.

Sendo assim, o objetivo deste estudo é investigar as evidências disponíveis na literatura científica nacional e internacional sobre a QVRS de mulheres com câncer de mama em tratamento radioterápico.

\section{MÉTODO}

Trata-se de uma revisão integrativa da literatura com as seguintes etapas percorridas: elaboração da questão de pesquisa, amostragem na literatura, avaliação dos estudos incluídos, categorização, interpretação e síntese dos resultados ${ }^{12}$. A questáo de pesquisa foi elaborada aplicando-se a estratégia, PICO em que "P" referiu-se à população do estudo (mulheres com câncer de mama); "I", à intervenção estudada ou à variável de interesse (radioterapia); “C”, à comparação com outra intervenção (não se aplicou neste estudo); e "O", ao desfecho de interesse (alteração da QVRS). Assim, a pergunta norteadora foi: quais são as evidências disponíveis na literatura científica nacional e internacional sobre a QVRS de mulheres com câncer de mama em tratamento radioterápico?

A busca dos estudos ocorreu no mês de agosto de 2020, nas bases eletrônicas de dados: CINAHL e MEDLINE (PubMed). As palavras-chave relacionadas com a temática foram selecionadas e verificadas nas bases de dados conforme a indexaçáo no MeSH Database foram: "quality of life", "women's health", "breast neoplams" "solid tumors" "radiotherapy"; "femininity"; "Chronic disease" "adverse effects" e o descritor nấo controlado "solid tumors" para a base de dados CINAHL. Para garantir a ampla busca dos artigos, foi utilizado o operador booleano AND e, como regra para os cruzamentos, quando a busca retornava mais de 200 artigos, introduziu-se um novo descritor para refiná-la ${ }^{13}$. Dessa forma, realizaram-se todas as combinaçóes possíveis entre os descritores em cada base de dados.

A seleção dos artigos ocorreu por meio da análise na íntegra por dois revisores independentes de acordo com os critérios de inclusão pré-definidos: artigos relacionados com a temática: QVRS de mulheres com câncer de mama em tratamento radioterápico disponíveis nos idiomas inglês, português e espanhol, e que abordassem o constructo de QV na forma geral: "a percepção do indivíduo de sua posição na vida, no contexto da cultura e sistemas de valores nos quais vive e em relação aos seus objetivos, expectativas, padróes e preocupaçóes" ${ }^{10}$, ou que englobassem um desses três aspectos: fadiga, sexualidade ou imagem corporal, considerando que geralmente são os domínios mais afetados durante o tratamento radioterápico. Foram excluídos os estudos do tipo relato de caso por apresentarem menor rigor metodológico.

A extração de dados dos estudos selecionados foi realizada por meio do instrumento de Nicolussi ${ }^{14}$, composto pelos seguintes itens: identificação da publicação, critérios de avaliação de estudos de QV e características metodológicas. Para a avaliação dos estudos e identificação da força de evidência, foi empregada a classificação proposta por Melnyk e Fineout-Overholt ${ }^{15}$.

\section{RESULTADOS}

Após a busca, foram encontrados 1.885 artigos, que resultaram em amostra final de 16 artigos. A Figura 1 ilustra o processo de seleção dos artigos desta revisão integrativa, de acordo com a metodologia do Preferred Reporting Items for Systematic Reviews and Meta-Analyses (PRISMA) ${ }^{16}$.

A caracterização dos 16 estudos incluídos na amostra encontra-se no (Quadro 1).

Todos os 16 estudos da amostra (Quadro 1) estão em língua inglesa. Entre os países onde a investigação foi realizada, destacaram-se os Estados Unidos, com 


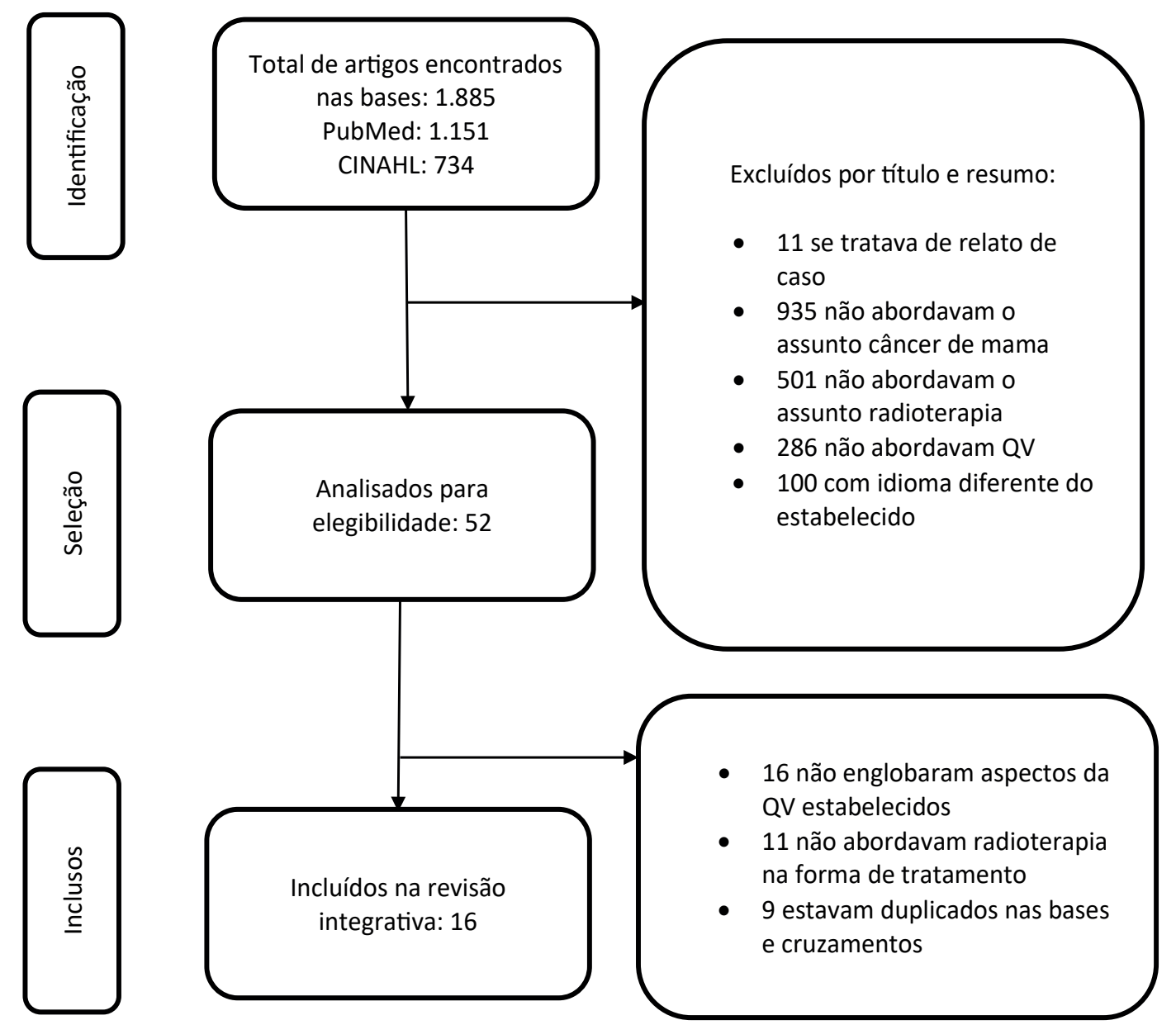

Figura 1. Fluxograma dos estudos incluídos na revisão integrativa de acordo com as bases de dados Legenda: $\mathrm{QV}=$ Qualidade de vida.

predominância de três $(18,8 \%)$ das pesquisas; seguido pelo Brasil com duas (12,5\%) das publicaçôes; e Polônia, Reino Unido, Egito, Singapura, Coréia, Canadá, Áustria, Turquia, Irã, Marrocos e Austrália apresentaram-se com um estudo, representando $11(68,7 \%)$ o restante da amostra. Foi possível observar que os estudos abrangeram todos os continentes, representando amplitude da importância da temática estudada.

Quanto ao ano de publicação, mesmo sem delimitação do período durante a seleção dos artigos incluídos, obtiveram-se estudos realizados entre 2004 e 2019, sendo as maiores concentraçóes nos anos de 2017 e 2018 $(18,8 \%)$ cada. Seis estudos $(37,5 \%)$ foram conduzidos nos anos de 2006, 2009 e 2012, sendo dois (12,5\%) em cada ano. Nos demais anos, houve divulgação de um artigo por ano, perfazendo os $25 \%$ restantes.

No tocante ao delineamento metodológico, seis (37,5\%) estudos eram do tipo observacional analítico transversal, dois $(12,5 \%)$ ensaios clínicos controlados e randomizados, dois de revisão sistemática e dois do tipo exploratório prospectivo, representando (25\%) da amostra, o restante (25\%) se tratava de um ensaio clínico não controlado e não randomizado, um estudo-piloto, uma revisão de literatura e um estudo de coorte.

Sobre o nível de evidência, quatro estudos apresentaram nível de evidência forte, sendo duas revisóes sistemáticas (nível I) e dois estudos com delineamento experimental do tipo ensaio clínico controlado e randomizado (nível II). Obteve-se um estudo de nível moderado (nível III), sendo um ensaio clínico bem delineado sem randomização. Apresentaram evidências fracas 11 estudos: três (nível IV) provenientes de estudo de coorte e caso controle bem delineados; e oito (nível VI) com evidências de um único estudo descritivo.

No que concerne aos objetivos dos estudos, notou-se que a maioria buscava investigar fatores determinantes de QVRS e focados em algum tipo de intervençáo para melhoria desta ou reduçáo dos sintomas. O Quadro 2 apresenta a síntese dos dados sobre objetivo, principais resultados e conclusóes dos artigos analisados. 
Quadro 1. Caracterização dos artigos analisados, Alfenas, Brasil, 2019

\begin{tabular}{|c|c|c|c|c|}
\hline Autores/Ano & Título & País & Idioma & NE \\
\hline $\begin{array}{l}\text { Senkus-Konefka e } \\
\text { Jassem, } 2006^{17}\end{array}$ & Complications of breast-cancer radiotherapy & Polônia & Inglês & $\mathrm{VI}$ \\
\hline $\begin{array}{l}\text { Gulluoglu et al., } \\
\qquad 2006^{18}\end{array}$ & $\begin{array}{l}\text { Factors related to post-treatment chronic pain in breast } \\
\text { cancer survivors: the interference of pain with life functions }\end{array}$ & Turquia & Inglês & IV \\
\hline $\begin{array}{l}\text { Alicikus et al., } \\
2009^{19}\end{array}$ & $\begin{array}{l}\text { Psychosexual and body image aspects of quality of life } \\
\text { in Turkish breast cancer patients: a comparison of breast } \\
\text { conserving treatment and mastectomy }\end{array}$ & $\begin{array}{l}\text { Reino } \\
\text { Unido }\end{array}$ & Inglês & VI \\
\hline Sbitti et al., $2011^{20}$ & $\begin{array}{l}\text { Breast cancer treatment and sexual dysfunction: Moroccan } \\
\text { women's perception }\end{array}$ & Marrocos & Inglês & IV \\
\hline $\begin{array}{l}\text { Alcântara-Silva et } \\
\text { al., } 2013^{21}\end{array}$ & $\begin{array}{l}\text { Fatigue related to radiotherapy for breast and/or } \\
\text { gynaecological cancer: a systematic review }\end{array}$ & Brasil & Inglês & I \\
\hline $\begin{array}{l}\text { Begovic-Juhant et } \\
\text { al., } 2012^{22}\end{array}$ & $\begin{array}{l}\text { Impact of body image on depression and quality of life } \\
\text { among women with breast cancer }\end{array}$ & EUA & Inglês & $\mathrm{VI}$ \\
\hline $\begin{array}{l}\text { Sundaresan et al., } \\
\qquad 2015^{23}\end{array}$ & $\begin{array}{l}\text { Patients' perceptions of health-related quality of life during } \\
\text { and after adjuvant radiotherapy for TINOMO breast cancer }\end{array}$ & Austrália & Inglês & $\mathrm{VI}$ \\
\hline $\begin{array}{l}\text { Shandiz et al., } \\
\qquad 2017^{24}\end{array}$ & $\begin{array}{l}\text { Investigating the quality of life and the related factors in } \\
\text { iranian women with breast cancer }\end{array}$ & Iran & Inglês & IV \\
\hline $\begin{array}{l}\text { Oberguggenberger } \\
\text { et al., } 2017^{25}\end{array}$ & $\begin{array}{l}\text { Self-reported sexual health: Breast cancer survivors } \\
\text { compared to women from the general population - an } \\
\text { observational study }\end{array}$ & Áustria & Inglês & $\mathrm{VI}$ \\
\hline Rim et al., $2017^{26}$ & $\begin{array}{l}\text { An assessment of quality of life for early phase after } \\
\text { adjuvant radiotherapy in breast cancer survivors: a Korean } \\
\text { multicenter survey (KROG 14-09) }\end{array}$ & Coréia & Inglês & III \\
\hline $\begin{array}{l}\text { Cheng et al., } \\
2018^{27}\end{array}$ & $\begin{array}{l}\text { Quality of life of elderly patients with solid tumours } \\
\text { undergoing adjuvant cancer therapy: a systematic review }\end{array}$ & Singapura & Inglês & I \\
\hline $\begin{array}{l}\text { Mortada et al., } \\
2018^{28}\end{array}$ & $\begin{array}{l}\text { Comparing health-related quality of life among breast } \\
\text { cancer patients receiving different plans of treatment, Egypt }\end{array}$ & Egito & Inglês & VI \\
\hline Cook et al., $2004^{29}$ & $\begin{array}{l}\text { Healing touch and quality of life in women receiving } \\
\text { radiation treatment for cancer: a randomized controlled } \\
\text { trial }\end{array}$ & EUA & Inglês & II \\
\hline $\begin{array}{l}\text { Sturgeon et al., } \\
2009^{30}\end{array}$ & $\begin{array}{l}\text { Effects of therapeutic massage on the quality of life among } \\
\text { patients with breast cancer during treatment }\end{array}$ & EUA & Inglês & VI \\
\hline $\begin{array}{l}\text { Alcântara-Silva et } \\
\text { al., } 2018^{31}\end{array}$ & $\begin{array}{l}\text { Music therapy reduces radiotherapy-induced fatigue in } \\
\text { patients with breast or gynecological cancer: a randomized } \\
\text { trial }\end{array}$ & Brasil & Inglês & II \\
\hline Abed et al., $2019^{32}$ & $\begin{array}{l}\text { Impact of self-reported exercise on recounted levels of } \\
\text { fatigue and anxiety in early-stage breast cancer radiation } \\
\text { therapy patients }\end{array}$ & Canadá & Inglês & VI \\
\hline
\end{tabular}

Legenda: $\mathrm{NE}=$ Nível de evidência.

\section{DISCUSSÃO}

Os assuntos abordados pelos artigos foram subdivididos em três categorias temáticas para melhor compreensão de seus resultados: "efeitos adversos da radioterapia", representando $(75 \%)$ do total; "terapias integrativas e complementares" (19\%); e "atividade física" (6\%). A totalidade das publicaçóes na língua inglesa pode demonstrar o interesse em tornar mais acessível o conhecimento produzido.

\section{CATEGORIA 1. EFEITOS ADVERSOS DA RADIOTERAPIA}

Os estudos incluídos nessa categoria abordaram os efeitos adversos decorrentes do tratamento radioterápico de maneira ampla e seu impacto na QVRS. Foram incluídos $12(75 \%)$ dos estudos analisados ${ }^{17-28}$, nos quais destaca-se que as mulheres que se submetem a esse tratamento estão susceptíveis a complicações somáticas: lesão cardíaca, pulmonar, complicaçóes no ombro e braço, malignidades secundárias ${ }^{17}$, complicaçôes mamárias ${ }^{23}$, comprometimento da sexualidade ${ }^{19,20,25,26}$ e de outros domínios da QVRS 
Quadro 2. Síntese dos dados sobre objetivo, principais resultados e conclusões dos artigos analisados, Alfenas, Brasil, 2019

\begin{tabular}{|c|c|c|c|}
\hline Referência & Objetivo & Resultado & Conclusão \\
\hline 17 & $\begin{array}{l}\text { Revisar o conhecimento } \\
\text { contemporâneo sobre a } \\
\text { toxicidade da radioterapia }\end{array}$ & $\begin{array}{l}\text { O tratamento radioterápico } \\
\text { pode estar relacionado a } \\
\text { uma série de complicações } \\
\text { somáticas que podem afetar a } \\
\text { QV do paciente e possivelmente } \\
\text { a sobrevida }\end{array}$ & $\begin{array}{l}\text { As mulheres submetidas à } \\
\text { radioterapia para câncer } \\
\text { de mama devem ter } \\
\text { acompanhamento, pois as } \\
\text { complicações tendem a aparecer } \\
\text { após longos períodos }\end{array}$ \\
\hline 18 & $\begin{array}{l}\text { Avaliar fatores } \\
\text { relacionados à dor crônica } \\
\text { pós-tratamento em } \\
\text { sobreviventes de câncer de } \\
\text { mama }\end{array}$ & $\begin{array}{l}\text { Foi descoberto que } \\
\text { a radioterapia está } \\
\text { significativamente relacionada } \\
\text { à dor crônica } p=0,049 \text {; OR: } \\
2,60 \text {; IC } 95 \% 1,07-6,30)\end{array}$ & $\begin{array}{l}\text { A frequência da dor no pós- } \\
\text {-tratamento é consideravelmente } \\
\text { maior em pacientes estavam } \\
\text { recebendo radioterapia }\end{array}$ \\
\hline 19 & $\begin{array}{l}\text { Avaliar os aspectos da } \\
\text { QV psicossexual e da } \\
\text { imagem corporal em } \\
\text { pacientes turcos com } \\
\text { câncer de mama: uma } \\
\text { comparação do tratamento } \\
\text { conservador da mama e } \\
\text { mastectomia }\end{array}$ & $\begin{array}{l}41 \% \text { dos pacientes sexualmente } \\
\text { ativos sofreram uma } \\
\text { deterioração do funcionamento } \\
\text { sexual após o tratamento }\end{array}$ & $\begin{array}{l}\text { Problemas psicossexuais e } \\
\text { de imagem corporal são } \\
\text { significativos e similares e } \\
\text { ocorrem em pacientes tratadas } \\
\text { para câncer de mama com } \\
\text { mastectomia ou tratamento } \\
\text { conservador }\end{array}$ \\
\hline 20 & $\begin{array}{l}\text { Avaliar prospectivamente a } \\
\text { escala da imagem corporal } \\
\text { e o impacto da terapia } \\
\text { do câncer de mama na } \\
\text { sexualidade das mulheres } \\
\text { de Marrocos }\end{array}$ & $\begin{array}{l}100 \% \text { dos indivíduos se } \\
\text { queixaram de uma deterioração } \\
\text { da sintomatologia após } \\
\text { os tratamentos e } 3 \% \text { das } \\
\text { disfunções sexuais foram } \\
\text { observadas após radioterapia }\end{array}$ & $\begin{array}{l}\text { O câncer de mama e seu } \\
\text { tratamento podem resultar em } \\
\text { dificuldades significativas no } \\
\text { funcionamento sexual e na vida } \\
\text { sexual }\end{array}$ \\
\hline 21 & $\begin{array}{l}\text { Avaliar o perfil, critérios de } \\
\text { avaliação e tratamentos } \\
\text { para fadiga relacionada } \\
\text { ao câncer mama e/ou } \\
\text { ginecológica em mulheres } \\
\text { submetidas à radioterapia }\end{array}$ & $\begin{array}{l}\text { O nível de fadiga pré- } \\
\text {-tratamento pode ser um } \\
\text { fator de risco importante, } \\
\text { sendo agravado durante a } \\
\text { radioterapia, reduzindo a } \mathrm{QV}\end{array}$ & $\begin{array}{l}\text { A detecção precoce da fadiga, } \\
\text { é relevante para propor } \\
\text { tratamentos adequados e obter } \\
\text { melhores condições clínicas, } \\
\text { adesão e continuidade do } \\
\text { tratamento radioterápico }\end{array}$ \\
\hline 22 & $\begin{array}{l}\text { Explorar a percepção } \\
\text { da imagem corporal, } \\
\text { atratividade física, e } \\
\text { feminilidade em um grupo } \\
\text { de mulheres com câncer } \\
\text { de mama }\end{array}$ & $\begin{array}{l}\text { Na percepção das mulheres } \\
\text { sobreviventes de câncer } \\
\text { de mama, o tratamento } \\
\text { radioterápico apresentou efeito } \\
\text { significativo na atratividade, } \\
\text { feminilidade e imagem corporal }\end{array}$ & $\begin{array}{l}\text { As percepções da imagem } \\
\text { corporal, atratividade e a } \\
\text { feminilidade aumentaram } \\
\text { a depressão e reduziram a } \\
\text { QV, especialmente durante o } \\
\text { primeiro ano de tratamento }\end{array}$ \\
\hline 23 & $\begin{array}{l}\text { Avaliar sintomas, funções } \\
\text { e atitudes pertinentes } \\
\text { durante e após a } \\
\text { radioterapia adjuvante } \\
\text { para câncer mama } \\
\text { T1NOMO e relacionar essas } \\
\text { classificações às avaliações } \\
\text { dos médicos de toxicidade }\end{array}$ & $\begin{array}{l}\text { A função física, cognitiva, social, } \\
\text { desempenho de papel, saúde } \\
\text { global e QV eram estáveis ou } \\
\text { deterioraram-se ligeiramente } \\
\text { durante o tratamento. Os } \\
\text { sintomas mais evidentes eram a } \\
\text { fadiga e problemas da mama }\end{array}$ & $\begin{array}{l}\text { A maioria dos aspectos da QVRS } \\
\text { piorou apenas ligeiramente } \\
\text { durante a radioterapia e } \\
\text { melhorou em alguns meses os } \\
\text { níveis basais }\end{array}$ \\
\hline 24 & $\begin{array}{l}\text { Investigar a QV e os } \\
\text { fatores relacionados em } \\
\text { mulheres iranianas com } \\
\text { câncer de mama }\end{array}$ & $\begin{array}{l}\text { A radioterapia teve um efeito } \\
\text { negativo na QV com alterações } \\
\text { nos domínios: insônia, fadiga, } \\
\text { função física e emocional }\end{array}$ & $\begin{array}{l}\text { O câncer de mama pode afetar } \\
\text { a QV das mulheres. Isso requer } \\
\text { mais atenção para encontrar } \\
\text { formas eficazes de promover e } \\
\text { melhorar a QV dessas pacientes }\end{array}$ \\
\hline 25 & $\begin{array}{l}\text { Investigar o resultado da } \\
\text { saúde sexual autorrelatado } \\
\text { de mulheres com câncer } \\
\text { de mama na rotina } \\
\text { pós-tratamento, em } \\
\text { comparação com mulheres } \\
\text { sem câncer de mama } \\
\text { anterior ou atual }\end{array}$ & $\begin{array}{l}\text { O câncer de mama e seus } \\
\text { tratamentos associados estão } \\
\text { frequentemente relacionados a } \\
\text { uma série de mudanças físicas } \\
\text { e emocionais, que podem } \\
\text { acarretar um impacto deletério } \\
\text { na saúde sexual }\end{array}$ & $\begin{array}{l}\text { Os resultados demonstram que } \\
\text { os problemas da saúde sexual } \\
\text { persistem na sobrevivência de } \\
\text { câncer de mama e diferem } \\
\text { significativamente da população } \\
\text { em geral }\end{array}$ \\
\hline
\end{tabular}


Quadro 2. continuação

\begin{tabular}{|c|c|c|c|}
\hline Referência & Objetivo & Resultado & Conclusão \\
\hline 26 & $\begin{array}{l}\text { Avaliar a QV dos } \\
\text { sobreviventes de câncer } \\
\text { de mama na fase inicial } \\
\text { crítica até três anos após a } \\
\text { conclusão da radioterapia } \\
\text { adjuvante }\end{array}$ & $\begin{array}{l}\text { Houve alteração da escala } \\
\text { EORTC QLQ-BR23 nas } \\
\text { áreas de sintomas como } \\
\text { dor, desconforto, ansiedade } \\
\text { e depressão; na escala de } \\
\text { função a sexualidade foi a mais } \\
\text { deteriorada }\end{array}$ & $\begin{array}{l}\text { As categorias dor/desconforto } \\
\text { e autocuidado e sintomas de } \\
\text { dor no braço, mama e imagem } \\
\text { corporal foram melhoradas, } \\
\text { enquanto ansiedade, depressão } \\
\text { e perspectiva futura não }\end{array}$ \\
\hline 27 & $\begin{array}{l}\text { Sintetizar as evidências de } \\
\text { QV durante e após terapia } \\
\text { adjuvante em pacientes } \\
\text { idosos com câncer }\end{array}$ & $\begin{array}{l}\text { Para pacientes idosas com } \\
\text { câncer de mama, não houve } \\
\text { mudança negativa e significativa } \\
\text { na QV global. Nenhum efeito } \\
\text { adverso duradouro na QV foi } \\
\text { observado após a conclusão do } \\
\text { tratamento adjuvante }\end{array}$ & $\begin{array}{l}\text { A QV durante e após a } \\
\text { quimioterapia adjuvante e/ } \\
\text { ou radioterapia é mantida ou } \\
\text { melhorada na maioria dos } \\
\text { pacientes idosos com tumores } \\
\text { sólidos }\end{array}$ \\
\hline 28 & $\begin{array}{l}\text { Medir a QV de } \\
\text { mulheres egípcias com } \\
\text { câncer de mama e } \\
\text { comparar as pontuações } \\
\text { QVRS em grupos de } \\
\text { pacientes que recebem } \\
\text { diferentes linhas de } \\
\text { tratamento }\end{array}$ & $\begin{array}{l}\text { Vários domínios da QV das } \\
\text { mulheres egípcias com câncer } \\
\text { de mama são diminuídos, } \\
\text { e o grupo de pacientes em } \\
\text { radioterapia mostra escore mais } \\
\text { baixo entre os subdomínios } \\
\text { da escala funcional EORTC } \\
\text { QLQ-C30 }\end{array}$ & $\begin{array}{l}\text { Os achados do estudo mostram } \\
\text { que a existência de outros } \\
\text { fatores como doenças crônicas } \\
\text { associadas e estágios avançados } \\
\text { do câncer podem interferir } \\
\text { na QV }\end{array}$ \\
\hline 29 & $\begin{array}{l}\text { Investigar influência do } \\
\text { toque terapêutico na QVRS } \\
\text { em mulheres que recebem } \\
\text { radioterapia para câncer } \\
\text { de mama }\end{array}$ & $\begin{array}{l}\text { Os indivíduos que receberam } \\
\text { o toque terapêutico durante } \\
\text { o tratamento radioterápico } \\
\text { demonstraram uma QVRS } \\
\text { melhor }\end{array}$ & $\begin{array}{l}\text { O toque terapêutico melhorou } \\
\text { a vitalidade, dor e função física } \\
\text { entre os itens da QVRS }\end{array}$ \\
\hline 30 & $\begin{array}{l}\text { Explorar o efeito de } \\
\text { massagem terapêutica } \\
\text { entre mulheres com câncer } \\
\text { de mama durante o } \\
\text { tratamento da radioterapia } \\
\text { e quimioterapia }\end{array}$ & $\begin{array}{l}\text { As participantes } \\
\text { experimentaram uma } \\
\text { redução em vários domínios } \\
\text { prejudicados da QV após } \\
3 \text { semanas de massagem } \\
\text { terapêutica }\end{array}$ & $\begin{array}{l}\text { A massagem terapêutica } \\
\text { mostrou benefícios na melhora } \\
\text { dos efeitos do tratamento, } \\
\text { reduzindo os efeitos colaterais } \\
\text { da quimioterapia e da } \\
\text { radioterapia e melhorando a QV }\end{array}$ \\
\hline 31 & $\begin{array}{l}\text { Estudar a influência da } \\
\text { musicoterapia na redução } \\
\text { da fadiga relacionada } \\
\text { ao câncer em mulheres } \\
\text { com câncer de mama ou } \\
\text { ginecológico durante o } \\
\text { tratamento radioterápico }\end{array}$ & $\begin{array}{l}\text { As mulheres relataram que } \\
\text { a musicoterapia fez diferença } \\
\text { em sua vida; tiveram uma } \\
\text { percepção positiva da } \\
\text { musicoterapia na melhora da } \\
\text { fadiga e redução do estresse }\end{array}$ & $\begin{array}{l}\text { A musicoterapia foi efetiva para } \\
\text { a redução da fadiga, sintomas } \\
\text { depressivos e na melhora da } Q V\end{array}$ \\
\hline 32 & $\begin{array}{l}\text { Investigar o impacto do } \\
\text { exercício autorrelato sobre } \\
\text { os níveis de fadiga e } \\
\text { ansiedade pré (TO), médio } \\
\text { (T1) e pós (T2) radioterapia } \\
\text { em pacientes em estágio } \\
\text { inicial do câncer de mama }\end{array}$ & $\begin{array}{l}\text { Os pacientes em estágio } \\
\text { inicial de câncer de mama } \\
\text { mostraram sinais de fadiga } \\
\text { e ansiedade, enquanto os } \\
\text { submetidos à terapia de } \\
\text { radiação independentemente } \\
\text { de exercício de autorrelato } \\
\text { medido por um questionário } \\
\text { atividade física }\end{array}$ & $\begin{array}{l}\text { O exercício pode reduzir a } \\
\text { ansiedade durante o tratamento } \\
\text { de radiação para pacientes } \\
\text { em estágio inicial de câncer de } \\
\text { mama }\end{array}$ \\
\hline
\end{tabular}

Legendas: QV = Qualidade de Vida; QVRS = Qualidade de Vida Relacionada à Saúde; EORTC QLQ = European Organization for Researchand Treatment of Cancer Quality of Life Questionnaire; $\mathrm{OR}=$ Odds ratio; IC = Intervalo de confiança.

(dor ${ }^{18,26}$ ansiedade, depressão e distúrbio do sono ${ }^{24,26}$, função emocional, função física ${ }^{24}$, fadiga ${ }^{21,23,26,28}$, e QV geral e global $^{27,28}$ ). Além de aspectos relacionados à atratividade, à feminilidade e à imagem corporal ${ }^{22}$.

De acordo com Oliveira et al. ${ }^{33}$, a lesão cardíaca descrita no estudo ${ }^{17}$ ocorre geralmente a longo prazo quando se irradia a mama do lado esquerdo. Podendo atingir qualquer estrutura cardíaca, incluindo pericárdio, miocárdio, valvas, sistema de condução e coronárias, acometendo cerca de $10 \%$ a $30 \%$ das mulheres $31,34,35$.

Quanto às complicaçóes pulmonares, estas podem ser precoces, entre elas, a pneumonite, ocorrendo de quatro a 12 semanas após o início do tratamento, tendo como sintomas principais: tosse seca, dispneia, febre baixa; e as complicações tardias, como a fibrose, que aparecem entre seis e 24 meses $^{17}$. 
$\mathrm{O}$ mesmo estudo ${ }^{17}$ verificou que as complicaçôes dos ombros e braço como sintomas de linfedema, plexopatia braquial e mobilidade do ombro prejudicada se configuram as mais problemáticas, podendo acometer até $90 \%$ das mulheres.

Essas mulheres também podem apresentar malignidades secundárias como câncer de mama contralateral e outros tumores na pele, endométrio, colorretal e pâncreas. O câncer de ovário, pulmão, renal, uterino, sarcoma e melanoma ocorrem com maior frequência. Entre as complicaçóes menos frequentes, podem ser citadas a fratura de costela, dor crônica, trombose venosa axilar e necrose óssea ${ }^{17}$.

Uma revisão integrativa corroborou os resultados dessa categoria, demonstrando que as mulheres em tratamento radioterápico podem apresentar complicações como restrição da movimentação, plexopatia braquial superior, danos ao coração e adicionalmente fibrose cutânea, espessamento endometrial, ganho de peso, diminuição do cálcio ósseo e dor ${ }^{33}$.

Os estudos ${ }^{19,20,26}$ apontaram alteraçóes na sexualidade, especialmente, deterioração da atividade sexual. Essa alteração tornou-se notória pela redução da libido, seguida pela perda de interesse no parceiro, insatisfação sexual, redução do prazer sexual, dispaureunia, secura vaginal e dificuldade em chegar ao orgasmo ${ }^{22}$, destacando que estes podem surgir logo após o início do tratamento radioterápico. Adicionalmente, um dos artigos analisado ${ }^{26}$ ressalta que, além da radioterapia, as alteraçóes na sexualidade também podem estar relacionadas com questôes culturais, considerando que a sexualidade, em algumas culturas, pode ser tratada como um tabu.

Da mesma forma, um estudo realizado no Irã verificou que as mulheres que receberam tratamentos adjuvantes conjuntamente, radioterapia e terapia hormonal, apresentaram uma porcentagem maior de disfunção sexual em comparaçáo com aquelas que receberam outros tratamentos adjuvantes ${ }^{36}$. Sebold et al..$^{37}$ reforçam que o declínio da atividade sexual decorrente do tratamento pode estar relacionado com a fadiga causada pela radioterapia e outros sintomas físicos específicos.

Ainda sobre a sexualidade, em sentido oposto, o estudo $^{25}$ não encontrou correlaçôes entre o tratamento radioterápico e a redução da atividade sexual. Corroborando este achado, o estudo da Verenhitach et al. ${ }^{38}$, que avaliou a sexualidade das mulheres com câncer e os fatores que interferem nela, evidenciou que a disfunção sexual não teve relação causal com a radioterapia.

É amplamente reconhecido que a sexualidade das mulheres pode ser particularmente complexa após o câncer de mama, ocorrendo mudanças sexuais bastante problemáticas. O impacto de tais mudanças tem potencial para durar vários anos após o término do tratamento, podendo estar associado aos graves efeitos adversos físicos e emocionais ${ }^{39}$.

Portanto, não se deve considerar isoladamente os efeitos do câncer de mama sobre a sexualidade das mulheres, é primordial identificar a associação com os efeitos físicos e psicossociais, que frequentemente são causados pelo tratamento e pelo processo patológico, o que influencia a QVRS ${ }^{38,40}$.

Em relação aos outros domínios da QVRS, a dor destacada pelos estudos ${ }^{18,26}$ é apontada na literatura como resultado da associação da radioterapia à cirurgia, podendo causar uma fibrose no local da cicatrização. Por esse motivo, o tratamento radioterápico pode ser citado como um fator predisponente para dor, sendo este também associado com as radiodermatites que ocorrem após a exposição à radiação ionizante, sendo um dos fatores que mais comprometem a QVRS?

A ansiedade, depressão e distúrbio de sono são sintomas apresentados pelo estudo ${ }^{26}$ como relacionados ao tratamento radioterápico. A ansiedade representa a incerteza relativa à doença e ao tratamento, além de ser um sintoma complexo e frequente ${ }^{41}$. A depressão pode estar associada à ativação de citocinas pró-inflamatórias, secundária à destruição dos tecidos ${ }^{42}$. E o distúrbio do sono sofre influência da dor e da depressão $0^{43}$.

A fadiga destacada nos estudos ${ }^{23,26,28}$ é um dos sintomas mais comuns e angustiantes experimentado por pacientes com câncer, causando comprometimento da $\mathrm{QV}^{44}$. Esta se configura como um sintoma de difícil manejo, pois seu processo fisiopatológico não é inteiramente conhecido. Diversos fatores estão relacionados com a fadiga, existem indícios de que a radioterapia gera o hipermetabolismo tumoral e os produtos gerados por esse metabolismo, em conjunto com o alto gasto energético, sejam os causadores desse sintoma ${ }^{9,45}$.

As alterações nos domínios desempenho de papel, autocuidado $^{28} \mathrm{e}$ função física ${ }^{24,28}$ são reflexos dos diversos sintomas supracitados vivenciados pela mulher durante o tratamento, uma vez que estes debilitam o estado físico e emocional, repercutindo diretamente na QVRS.

$\mathrm{O}$ estudo ${ }^{27}$ não demonstrou correlação entre o tratamento radioterápico e QV geral, todavia um outro, produzido por Nicolussi e Sawada ${ }^{46}$, evidenciou que as pacientes que receberam radioterapia apresentaram pior escore dos domínios de QV geral e global. Esse dado corroborou o estudo ${ }^{28}$ que mostrou que a QV global e a geral dos pacientes que estavam em tratamento radioterápico tiveram mais alteraçôes.

Uma pesquisa ${ }^{22}$ demonstrou que, na percepção das mulheres sobreviventes de câncer de mama, o tratamento radioterápico não apresentou efeito significativo na 
atratividade, feminilidade e imagem corporal. Todavia, em um estudo de revisão de literatura realizado por Rezaei et al. ${ }^{47}$ sobre os fatores que influenciam a imagem corporal de mulheres com câncer de mama, foi evidenciado que os efeitos residuais da radioterapia, como mudança na coloraçáo da pele do local irradiado, dor crônica e linfedema, são fatores que afetam significativamente a percepção das mulheres em relação à sua imagem corporal.

Os achados da presente categoria demonstraram a importância de uma avaliação holística do paciente desde o diagnóstico, durante o tratamento e ao longo da vida, uma vez que os estudos evidenciaram que alguns efeitos tendem a aparecer após longos períodos de latência, podendo acarretar danos progressivos. Medir a QVRS permite avaliar o impacto do tratamento na percepção do indivíduo sobre sua vida e o quanto as alteraçóes decorrentes da doença podem interferir em seus domínios ${ }^{46}$.

\section{CATEGORIA 2. TERAPIAS INTEGRATIVAS E COMPLEMENTARES}

Essa categoria englobou três estudos ${ }^{29-31}$ (19\%) dos artigos analisados, estes salientam as terapias integrativas e complementares como musicoterapia, massagem terapêutica e Reiki na melhora dos sintomas decorrentes do tratamento radioterápico.

Nas últimas décadas, a medicina complementar e alternativa vem sendo utilizada como auxílio no tratamento de neoplasias com enfoque na redução dos sintomas, sendo composta por abordagens de cuidado e recursos terapêuticos que possuem um importante papel na saúde global ${ }^{48}$.

Com relação à musicoterapia, o estudo ${ }^{31}$ constatou que esta é uma estratégia eficaz na redução da fadiga, após realizar sessóes individuais de 30 a 40 minutos com músicas clássicas, românticas e barrocas escolhidas pela pesquisadora, sendo realizada duas vezes por semana antes das sessôes de radioterapia. Um estudo desenvolvido em Taiwan também mostrou eficácia da música sobre a dor e fadiga, após realizar cinco sessóes de 30 minutos, cinco vezes na semana, com músicas clássicas, típicas de Taiwan, religiosas e de salāo $0^{49}$.

$\mathrm{O}$ estudo $^{30}$ mostrou que a massagem terapêutica (massagem sueca com toques leves e rítmicos, evoluindo para toques mais firmes) aplicada uma vez na semana, por três semanas consecutivas, com duração de 30 minutos cada sessão, apresentou redução dos efeitos colaterais e melhora da percepção da QVRS. Uma amostra randomizada cuja massagem terapêutica foi realizada por cinco semanas consecutivas, três vezes na semana, com duraçáo de 30 minutos cada sessáo, apresentou uma melhora nos sintomas de ansiedade, depressão, transtornos do humor, incluindo raiva, afeto positivo e redução de sintomas físicos como dor e fadiga ${ }^{50}$.
O toque terapêutico (Reiki), que se demonstrou eficaz na melhora da QVRS (nos domínios de vitalidade, dor e função física) quando aplicado em mulheres com câncer de mama em tratamento radioterápico, foi desenvolvido em três etapas, sendo a primeira sessão após terem completado um terço do tratamento, quatro sessóes no meio do período do tratamento, e a última sessão depois da finalização o tratamento, cada sessão do toque terapêutico teve duração de aproximadamente 30 minutos $^{29}$.

Já o estudo de revisão de literatura realizado por Tabatabaee et al. ${ }^{51}$ mostrou que o toque terapêutico, com duração de 20 a 40 minutos a cada sessão, é benéfico na melhora do humor, bem-estar, redução da dor, náusea e vômito, relaxamento, elevação espiritual, melhora do sono e aumento da capacidade funcional. No entanto, em um estudo de coorte desenvolvido por Younus et al..$^{52}$, o toque terapêutico aplicado por cinco semanas consecutivas, após as sessôes de radioterapia com frequência de três vezes na semana e duração de 15 a 20 minutos, não demonstrou melhora nos aspectos da QVRS (fadiga e humor).

É oportuno destacar que as terapias integrativas são intervenções não invasivas, com mínimos efeitos colaterais, baixo custo e podendo ser aplicadas por um profissional treinado, no tratamento humanizado e integral do paciente com câncer.

\section{CATEgoRIA 3. ATIVIDADE FísICA}

Nessa categoria, foi incluído um estudo (6\%) que relaciona a prática de atividade física na redução dos sintomas de ansiedade e fadiga decorrentes do tratamento, os resultados desta pesquisa apontaram que a atividade física ativa demonstrou melhora somente nos sintomas de ansiedade, sendo utilizado o questionário International Physical Activity Questionnaire, cuja atividade física estava ligada ao cotidiano, trabalho, atividades recreativas ou exercícios regulares nos últimos sete dias. Ressaltando ainda que foram considerados ativos os pacientes que realizaram atividades que requerem mais esforço físico e que provocaram hiperventilação ${ }^{32}$.

Há evidências de que a prática da atividade física aumenta a sensação de energia ocasionando diminuição da percepção da fadiga, sendo considerada uma modalidade terapêutica adjuvante no enfrentamento e reabilitaçãa ${ }^{53,54}$. Desta forma, mostrou-se eficaz sobre os domínios de ansiedade e fadiga de mulheres com câncer de mama que estavam em tratamento radioterápico e a mais satisfatória na redução dos níveis de ansiedade ${ }^{55}$. Vale ressaltar que nenhum desses estudos abordou a prática de atividade física da mesma maneira que o estudo incluído nessa categoria.

A atividade física não está ligada apenas à prática de esportes, mas à prática regular de atividades físicas 
cotidianas, o que pode atuar fortemente na redução dos níveis de ansiedade durante o tratamento adjuvante, sendo uma alternativa aos pacientes que estão sendo submetidos a esse tipo de terapia.

As limitaçóes desta pesquisa se deram em relação ao baixo nível de evidência, em que 11 estudos apresentaram evidências fracas, o que implica em vieses nos resultados. Sugere-se o desenvolvimento de novos estudos com melhor nível de evidência e com delineamentos mais rigorosos para comprovar a efetividade das intervençóes e a disseminação de práticas que interferem na QVRS de pacientes oncológicos, no intuito de garantir a translaçáo do conhecimento e a excelência da assistência prestada a essa população.

\section{CONCLUSÃO}

A presente revisão incluiu artigos de todos os continentes, demonstrando amplitude e relevância de estudos sobre a QV de mulheres em tratamento radioterápico. Os estudos analisados abordaram os efeitos colaterais do tratamento radioterápico e apresentaram evidências de práticas integrativas e complementares efetivas para amenizá-los e consequentemente melhorar a QVRS. Espera-se que a síntese dos estudos analisados forneça subsídios para a melhoria da prática clínica dos profissionais que prestam assistência às mulheres com câncer de mama em tratamento radioterápico e consequentemente tenham um impacto positivo na sua QVRS.

\section{CONTRIBUIÇÕES}

Todos os autores contribuíram substancialmente na concepção e no planejamento do estudo; na obtenção, análise e/ ou interpretação dos dados; assim como na redação e/ou revisão crítica; e aprovaram a versão final a ser publicada.

\section{DECLARAÇÃO DE CONFLITO DE INTERESSES}

Nada a declarar.

\section{FONTES DE FINANCIAMENTO}

Coordenação de Aperfeiçoamento de Pessoal de Nível Superior (Capes) - Brasil. Código de Financiamento 001.

\section{REFERÊNCIAS}

1. Cao B, Soerjomataram I, Bray F, et al. The burden and prevention of premature deaths from noncommunicable diseases, including cancer: a global perspective. In: Wild CP, Weiderpass E, Stewart BW, editors. World Cancer
Report: cancer research for cancer prevention. Lyon: International Agency for Research on Cancer; 2020. p. 16-22.

2. Marta GN, Hanna SA, Martella E, et al. Câncer de mama estádio inicial e radioterapia: atualização. Rev Assoc Med Bras. 2011;57(4):468-74. doi: https://doi.org/10.1590/ S0104-42302011000400024

3. Instituto Nacional de Câncer José Alencar Gomes da Silva. Estimativa 2020: incidência de câncer no Brasil [Internet]. Rio de Janeiro: INCA; 2019 [acesso 2020 jul 9]. Disponível em: https://www.inca.gov.br/sites/ufu. sti.inca.local/files//media/document//estimativa-2020incidencia-de-cancer-no-brasil.pdf

4. Miller KD, Nogueira L, Mariotto AB, et al. Cancer treatment and survivorship statistics, 2019. CA Cancer J Clin. 2019;69(5):363-85. doi: https://doi.org/10.3322/ caac. 21565

5. Andersen BL, Karlsson JA, Anderson B, et al. Anxiety and cancer treatment: response to stressful radiotherapy. Health Psychol. 1984;3(6):535-51. doi: https://doi. org/10.1037//0278-6133.3.6.535

6. Silva ECS, Silva JM, Silva LF, et al. Câncer de mama e qualidade de vida durante o tratamento radioterápico. Ciênc Biol Saúde [Internet]. 2014 [acesso 2020 set 9];1(3):85-93. Disponível em: https://core.ac.uk/ download/pdf/230436701.pdf

7. Sucala M, Schnur JB, Brackman E, et al. The role of specific and core dysfunctional beliefs in breast cancer radiotherapy patients' fatigue. J Health Psychol. 2014;19(8):957-65. doi: https://doi.org/10.1177/1359105313482166

8. Bahia JC, Lima CM, Oliveira MM, et al. Fadiga em mulheres com câncer de mama submetidas à radioterapia. Rev Bras Cancerol. 2019;65(2):e-09089. doi: https://doi. org/10.32635/2176-9745.RBC.2019v65n2.89

9. Rocha DM, Pedrosa AO, Oliveira AC, et al. Evidências científicas sobre os fatores associados à qualidade de vida de pacientes com radiodermatite. Rev Gaúcha Enferm. 2018;39:e2017-0224. doi: https://doi. org/10.1590/1983-1447.2018.2017-0224

10. The Whoqol Group. The World Health Organization quality of life assessment (WHOQOL): position paper from the World Health Organization. Soc Sci Med. 1995;41(10):1403-9. doi: http://doi.org/10.1016/02779536(95)00112-k

11. Ferrel BF, Dow KH, Grant M. Measurement of the quality of life in cancer survivors. Qual Life Res.1995;4(6):52331. doi: http://doi.org/10.1007/BF00634747

12. Mendes KDS, Silveira RCCP, Galvão CM. Revisão integrativa: método de pesquisa para a incorporação de evidências na saúde e na enfermagem. Texto Contexto Enferm. 2008;17(4):758-64. doi: http://doi. org/10.1590/S0104-07072008000400018

13. Lopes CMM. Escala de avaliação de risco para o desenvolvimento de lesóes decorrentes do posicionamento 
cirúrgico: construção e validação [tese na Internet]. Ribeirão Preto, SP: Universidade de São Paulo; 2014. doi: http://doi.org/10.11606/T.22.2014.tde-21052014184456

14. Nicolussi AC. Qualidade de vida de pacientes com câncer de cólon e reto: revisão integrativa da literatura [dissertação na Internet]. Ribeirão Preto, SP: Universidade de São Paulo; 2008. doi: http://doi.org/10.11606/D.22.2008. tde-03092008-111111

15. Melnyk BM, Fineout-Overholt E. Evidence-based practice in nursing \& healthcare: a guide to best practice. 4th ed. Philadelphia: Wolters Kluwer Health; 2019.

16. Galvão TF, Panssani TSA, Harrad D. Principais itens para relatar revisóes sistemáticas e meta-análises: a recomendação PRISMA [tradução]. Epidemiol Serv Saúde. 2015;24(2):335-42. doi: https://doi.org/10.5123/ S1679-49742015000200017

17. Senkus-Konefka E, Jassem J. Complications of breastcancer radiotherapy. Clin Oncol (R Coll Radiol). 2006;18(3):229-35. doi: https://doi.org/10.1016/j. clon.2005.11.004

18. Gulluoglu BM, Cingi A, Cakir T, et al. Factors related to post-treatment chronic pain in breast cancer survivors: the interference of pain with life functions. Int J Fertil Womens Med [Internet]. 2006 [cited 2020 Sept 9];51(2):75-82. Available from: https://europepmc.org/ article/med/16881383

19. Alicikus ZA, Gorken IB, Sen RC, et al. Psychosexual and body image aspects of quality of life in turkish breast cancer patients: a comparison of breast conserving treatment and mastectomy. Tumori. 2009;95(2):212-8. doi: https://doi.org/10.1177/030089160909500213

20. Sbitti Y, Kadiri H, Essaidi I, et al. Breast cancer treatment and sexual dysfunction: Moroccan women's perception. BMC Womens Health. 2011;11:29. doi: http://doi. org/10.1186/1472-6874-11-29

21. Alcântara-Silva TRM, Freitas-Junior R, Freitas NMA, et al. Fatigue related to radiotherapy for breast and/or gynaecological cancer: a systematic review. J Clin Nurs. 2013;22(19-20):2679-86. doi: http://doi.org/10.1111/ jocn. 12236

22. Begovic-Juhant A, Chmielewski A, Iwuagwu $S$, et al. Impact of body image on depression and quality of life among women with breast cancer. J Psychosoc Oncol. 2012;30(4):446-60. doi: http://doi.org/10.1080/0734 7332.2012 .684856

23. Sundaresan P, Sullivan L, Pendlebury S, et al. Patients' perceptions of health-related quality of life during and after adjuvant radiotherapy for T1N0M0 breast cancer. Clin Oncol (R Coll Radiol). 2015;27(1):9-15. doi: http://doi.org/10.1016/j.clon.2014.09.007

24. Shandiz FH, Karimi FZ, Anbaran ZK, et al. Investigating the quality of life and the related factors in iranian women with breast cancer. Asian Pac J Cancer Prev.
2017;18(8):2089-92. doi: http://doi.org/10.22034/ APJCP.2017.18.8.2089

25. Oberguggenberger A, Martini C, Huber N, et al. Selfreported sexual health: breast cancer survivors compared to women from the general population - an observational study. BMC Cancer. 2017;17(1):599. doi: http://doi. org/10.1186/s12885-017-3580-2

26. Rim CH, Ahn SJ, Kim JH, et al. An assessment of quality of life for early phase after adjuvant radiotherapy in breast cancer survivors: a Korean multicenter survey (KROG 14-09). Health Qual Life Outcomes. 2017;15(1):96. doi: http://doi.org/10.1186/s12955-017-0673-1

27. Cheng KKF, Lim EYT, Kanesvaran R. Quality of life of elderly patients with solid tumours undergoing adjuvant cancer therapy: a systematic review. BMJ Open. 2018;8(1):e018101. doi: http://doi.org/10.1136/ bmjopen-2017-018101

28. Mortada EM, Salem RA, Elseifi OS, et al. Comparing health-related quality of life among breast cancer patients receiving different plans of treatment, Egypt. J Community Health. 2018;43(6):1183-91. doi: http:// doi.org/ 10.1007/s10900-018-0538-5

29. Cook CAL, Guerrerio JF, Slater VE. Healing touch and quality of life in women receiving radiation treatment for cancer: a randomized controlled trial. Alter Ther Health Med. 2004;10(3):34-41.

30. Sturgeon M, Wetta-Hall, Hart T, et al. Effects of therapeutic massage on the quality of life among patients with breast cancer during treatment. J Altern Complement Med. 2009;15(4):373-80. doi: https://doi. org/10.1089/acm.2008.0399

31. Alcântara-Silva TR, Freitas-Junior R, Freitas NMA. Music therapy reduces radiotherapy-induced fatigue in patients with breast or gynecological cancer: a randomized trial. Integr Cancer Ther. 2018;17(3):62835. doi: https://doi.org/10.1177/1534735418757349

32. Abed J, Dolan L, Jones J, et al. Impact of self-reported exercise on recounted levels of fatigue and anxiety in early-stage breast cancer radiation therapy patients. J Med Imaging Radiat Sci. 2019;50(2):227-33. doi: https://doi. org/10.1016/j.jmir.2018.12.001

33. Oliveira KFP, Lima LDAC, Carvalho SML. Morbidades após tratamento do câncer de mama. An Fac Med Olinda [Internet]. 2018 [acesso 2020 set 9];2(2):91-95. Disponível em: https://afmo.emnuvens.com.br/afmo/ article/view/43/40

34. Donnellan E, Phelan D, McCarthy CP, et al. Radiationinduced heart disease: a practical guide to diagnosis and management. Cleve Clin J Med. 2016;83(12):914-22. doi: http://doi.org/10.3949/ccjm.83a.15104

35. Bittar CS, Fonseca SMR. Radioterapia e cardiotoxicidade. Rev Soc Cardiol Estado de São Paulo. 2017;27(4):274-7. doi: http://doi.org/10.29381/01038559/20172704274-7 
36. Cobo-Cuenca AI, Martín-Espinosa NM, SampietroCrespo A, et al. Sexual dysfunction in Spanish women with breast cancer. Plos One. 2018;13(8):e0203151. doi: https://doi.org/10.1371/journal.pone.0203151

37. Sebold N, Laverde AG, Rosa LM, et al. Sexualidade no enfrentamento do câncer de mama: estratégias de superação. Rev Recien. 2016;6(18):51-62. doi: https:// doi.org/10.24276/rrecien2358-3088.2016.6.18.51-62

38. Verenhitach BD, Medeiros JN, Elias S, et al. Câncer de mama e seus efeitos sobre a sexualidade: uma revisão sistemática sobre abordagem e tratamento. Femina [Internet]. 2014 [acesso 2020 set 7];42(1):4-9. Disponível em: http://files.bvs.br/upload/S/0100-7254/2014/ v42n1/a4806.pdf

39. Emilee G, Ussher JM, Perz J. Sexuality after breast: a review. Maturitas. 2010;66(4):397-407. doi: https://doi. org/10.1016/j.Maturitas.2010.03.027

40. Hammerschmidt KSA, Rosa LM, Alvarez AM et al. Comportamento sexual das mulheres em tratamento radioterápico. Ciênc Cuid Saúde. 2016;15(1):194201. doi: https://doi.org/10.4025/cienccuidsaude. v15i1.25064

41. Stamm B, Girardon-Perlini NMO, Pasqualoto AS, et al. Intervenção telefônica para manejo da ansiedade de pacientes oncológicos: ensaio clínico randomizado. Acta Paul Enferm. 2018;31(2):137-43. doi: https://doi. org/10.1590/1982-0194201800021

42. Raison CL, Demetrashvili M, Capuron L, et al. Neuropsychiatric adverse effects of interferon-alpha: recognition and management. CNS Drugs. 2005;19(2):10523. doi: http://doi.org/10.2165/00023210-20051902000002

43. Mansano-Schlosser TC, Ceolim MF. Fatores associados à má qualidade do sono em mulheres com câncer de mama. Rev Latino-Am Enfermagem. 2017;25:e2858. doi: http://doi.org/10.1590/1518-8345.1478.2858

44. Bower JE, Wiley J, Petersen L, et al. Fatigue after breast cancer treatment: biobehavioral predictors of fatigue trajectories. Health Psychol. 2018;37(11):1025-34. doi: http://doi.org/10.1037/hea0000652

45. Madden J, Newton S. Why am I so tired all the time? Understanding cancer-related fatigue. Clin J Oncol Nurs. 2006;10(5):659-61. doi: http://doi.org/10.1188/06. CJON.659-661

46. Nicolussi AC, Sawada NO. Qualidade de vida de pacientes com câncer de mama em terapia adjuvante. Rev Gaúcha Enferm. 2011;32(4):759-66. doi: http:// doi.org/10.1590/S1983-14472011000400017

47. Rezaei M, Elyasi F, Janbabai G, et al. Factors influencing body image in women with breast cancer: a comprehensive literature review. Iran Red Crescent Med J. 2016;18(10):e39465. doi: https://doi.org/10.5812/ircmj.39465

48. Carlson LE, Zelinski E, Toivonen K. Mind-body therapies in cancer: what is the latest evidence? Curr Oncol Rep.
2017;19(10):67. doi: https://doi.org/10.1007/s11912017-0626-1

49. Hsieh FC, Miao NF, Tseng IJ, et al. Effect of homebased music intervention versus ambient music on breast cancer survivors in the community: a feasibility study in Taiwan. Eur J Cancer Care (Engl). 2019;28(4):e13064. doi: https://doi.org/10.1111/ecc.13064

50. Darabpour S, Kheirkhah M, Ghasemi E. Effects of swedish massage on the improvement of mood disorders in women with breast cancer undergoing radiotherapy. Iran Red Crescent Med J. 2016;18(11):e25461. doi: https://doi.org/10.5812/ircmj.25461

51. Tabatabaee A, Tafreshi MZ, Rassouli M, et al. Effect of therapeutic touch in patients with cancer: a literature review. Med Arch. 2016;70(2):142-7. doi: https://doi. org/10.5455/medarh.2016.70.142-147

52. Younus J, Lock M, Vujovic O, et al. A case-control, monocenter, open-label, pilot study to evaluate the feasibility of therapeutic touch in preventing radiation dermatitis in women with breast cancer receiving adjuvant radiation therapy. Complement Ther Med. 2015;23(4):612-6. doi: https://doi.org/10.1016/j.ctim.2014.11.003

53. Rodrigues LF. A redução da fadiga oncológica através do exercício físico. Rev Bras Fisiol Exerc. 2019;18(1):57-7. doi: https://doi.org/10.33233/rbfe.v18i1.2879

54. Maqbali M, Hughes C, Dunwoody L, et al. Exercise interventions to manage fatigue in women with gynecologic cancer: a systematic review. Oncol Nurs Forum. 2019;46(1):71-82. doi: https://doi. org/10.1188/19.ONF.71-82

55. Saço LF, Cunha CFB, Silva RA, et al. Ansiedade em mulheres com câncer de mama e sua relação com a atividade física. HU Rev [Internet]. 2014 [acesso 2020 set 23];38(3):187-192. Disponível em: https://periodicos. uff.br/index.php/hurevista/article/view/2050

Recebido em 17/11/2020 Aprovado em 26/3/2021 